\title{
ESPACIALIZAÇÃO DAS DESCARGAS ELÉTRICAS ATMOSFÉRICAS NO ESTADO DE SÃO PAULO PARA OS ANOS DE 2005-2006, E SUAS CONSEQUÊNCIAS
}

\author{
CONCEIÇÃO, Rodrigo Pucci da - rodrigopucci@yahoo.com.br \\ Mestrando Bolsista CAPES do Programa de Pós-Graduação em Geografia, Universidade \\ Estadual Paulista, Campus de Rio Claro, São Paulo \\ ANDRÉ, Iára Regina Nocentini - iaranocentini@gmail.com \\ Professor Assistente, Departamento de Geografia, Universidade Estadual Paulista, \\ Campus de Rio Claro, São Paulo.
}

\author{
AZEVEDO, Thiago Salomão de - thiagosalomaodeazevedo@gmail.com \\ Professor Assistente, Faculdades Integradas Claretianas de Rio Claro, São Paulo
}

\begin{abstract}
RESUMO: Os fenômenos atmosféricos denominados descargas elétricas são complexos e representam prejuízos socioeconômicos e ambientais. A distribuição de ocorrências deste fenômeno pelo planeta é desigual, sendo que algumas regiões apresentam números consideráveis de registros. Esta diferença é visível em escalas maiores, como no Estado de São Paulo, por exemplo, que apresenta grande diversidade na quantidade de descargas elétricas registradas em seu território. Além dos fatores climáticos, as características do relevo e o fator urbanização, aparentemente, também influenciam na diferença de ocorrências. Devido a importância deste fenômeno para a sociedade, e a pequena quantidade de estudos geográficos sobre o assunto, escolheu-se por realizar o mapeamento deste sinistro para o estado, analisando tanto o fenômeno, quanto as vítimas registradas entre os anos de 2005-2006.
\end{abstract}

Palavras-chave: descargas elétricas atmosféricas, Estado de São Paulo, sistema de informação geográfica, vítimas fatais e feridos.

SPATIALIZATION OF ATMOSPHERIC ELECTRICAL DISCHARGES IN SÃO PAULO STATE FOR THE YEAR 2005-2006, AND ITS CONSEQUENCES

ABSTRACT: The atmospheric events denominated electric discharges are complexes and represent social, economic and environmental damages. The distribution of occurrences of this phenomenon the planet is uneven, and some regions have considerable numbers of records. This difference is visible at larger scales, as the State of São Paulo, for example, that presents great diversity in the amount of electrical discharges registered in its territory. Besides climatic factors, the characteristics of topography and urbanization factor apparently also influence the difference in occurrences. Given the importance of this phenomenon for society, and the small amount of geographical studies on the subject, was chosen to perform the mapping of this sinister to the state, analyzing both the phenomenon, as the victims recorded between the years 2005-2006.

Key-words: atmospheric electrical discharges, State of São Paulo, geographic information system, wounded and lethal victims.

\section{INTRODUÇÃO}

Os fatores climáticos são determinantes na organização do espaço, principalmente à medida que a sociedade depende diretamente da sua variabilidade e dos riscos e impactos gerados por estes.

Os fenômenos atmosféricos de grande intensidade estão associados a dinâmicas climáticas próprias, geradas naturalmente. Marcelino (2003) afirma que os fenômenos tem relação com sua origem, agrupando-os nas formas meteóricas hídricas (chuvas, neve e nevoeiro), mecânicas (tornados) e elétricas (raios e relâmpagos).

As descargas elétricas atmosféricas ou trovoadas são caracterizadas pela ocorrência de raios, relâmpagos (luz que resulta da incandescência do ar) e de 
trovões (som que resulta da expansão brusca do ar). Geralmente, a trovoada está associada à precipitação no estado líquido (chuva) ou no estado sólido (granizo) (SOUZA, 2002; PORTUGAL, 2009).

O raio ocorre quando duas partículas com cargas elétricas de sinais opostos são fortemente atraídas uma para a outra. Contudo, o ar é fraco condutor da corrente elétrica, não permitindo que estas se aproximem. Quando o ar que se encontra entre as cargas não consegue impedir a sua aproximação, dá-se uma descarga elétrica denominada de raio. Já o relâmpago ocorre da incandescência do ar aquecido à passagem do raio na coluna de ar, resultante da conversão da energia eletromagnética em energia calorífica, podendo apresentar carga positiva e negativa. Entretanto, ao longo da trajetória do raio, através da coluna de ar, com diâmetro de poucos centímetros, ocorre uma expansão brusca do ar aquecido que origina uma onda de choque que dá origem a um ruído característico chamado de trovão, que é um subproduto do raio (FORSDYKE, 1975).

Desde a descoberta da natureza elétrica dos relâmpagos pelo cientista americano Benjamim Franklin (1706-1790) até os dias atuais, a pesquisa de descargas atmosféricas e seus fenômenos associados apresentou avanços significativos com o aprimoramento de novas tecnologias de medição (NACCARATO, 2006). Técnicas e instrumentos de medição como osciloscópios de alta resolução e com grande velocidade de captação, câmeras de vídeo ultrarrápidas com até 8000 quadros/s, satélites com sensores ópticos e redes intercontinentais de sensores eletromagnéticos permitiram o esclarecimento de muitas dúvidas relacionadas aos fenômenos elétricos da troposfera e estratosfera.

Contudo, muito pouco se sabe sobre os processos para a ocorrência das descargas elétricas atmosféricas. Quanto mais as tecnologias de medição são aprimoradas, mais particularidades desses fenômenos são descobertas, obrigando a ciência a buscar constantemente a correlação entre os diferentes processos físicos e químicos que ocorrem na troposfera para encontrar explicações, ou poder elaborar hipóteses, sobre este fenômeno atmosférico (NACCARATO, 2006).

A grande maioria dos relâmpagos ocorre no céu, sem o contato com a superfície da Terra, porém a parte que atinge o solo é numerosa o suficiente para causar danos consideráveis nas estruturas construídas pelo homem, e principalmente à saúde da sociedade, sendo considerada a segunda maior causa de morte por fenômenos meteorológicos, de acordo com dados da Cruz Vermelha (NACCARATO, 2006).

No Brasil, os estudos climáticos sobre os raios são recentes e as primeiras análises são dos anos de 1990. Até este período, não havia uma rede de detecção de raios abrangente no país dificultando as pesquisas sobre este fenômeno atmosférico (NACCARATO, 2006). Contudo, as relações entre a frequência de descargas elétricas atmosféricas e a ocorrência de vítimas (mortes e feridos) decorrentes deste fenômeno ainda é pouco pesquisada. Neste trabalho foi realizado o levantamento bibliográfico sobre o tema, o 
mapeamento e análises deste fenômeno, e de vítimas fatais e/ou feridos que aconteceram no Estado de São Paulo, entre os anos de 2005 e 2006.

\section{TEMPESTADES SEVERAS E DESCARGAS \\ ELÉTRICAS ATMOSFÉRICAS}

As tempestades são habitualmente constituídas por nuvens eletrificadas do tipo Cumulonimbus, que apresentam trovões e relâmpagos. Nelas ocorrem processos de geração e separação de cargas elétricas que dão origem as descargas elétricas atmosféricas.

Uma nuvem de tempestade geralmente apresenta diâmetro e extensão vertical em torno de 10 a $20 \mathrm{~km}$, e pode movimentar-se com velocidade de 40 a 50 $\mathrm{km} / \mathrm{h}$, apresentando tempo de duração em média de 30 a 90 minutos.

Essas tempestades são conhecidas pelos meteorologistas como complexos convectivos de mesoescala; geralmente, são tempestades severas, apresentam altas taxas de precipitação, ventos intensos e eventualmente produzem granizo (PINTO Jr. E PINTO, 2000; GOMES, 2003; SILVA DIAS, 1987).

Os complexos convectivos de mesoescala são sistemas quase circulares, com inúmeras tempestades interligadas, possuem duração de aproximadamente 10 a 12 horas e diâmetros de 300 a $400 \mathrm{~km}$. Vários agrupamentos de tempestades em escala sinótica podem dar origem às tempestades tropicais e extratropicais ou ciclones, que atingem de centenas a milhares de quilômetros, com ventos superiores a $300 \mathrm{~km} / \mathrm{h}$ e duração de vários dias (GOMES, 2003; PINTO Jr. E PINTO, 2000; LIMA, 2005; SILVA DIAS, 1987).

A altura dos topos das nuvens de tempestade depende da latitude geográfica. Desta forma, a expansão vertical de uma tempestade raramente ultrapassa 8 $\mathrm{km}$ de altitude nas altas latitudes, já nas baixas latitudes podem alcançar até $20 \mathrm{~km}$ de altitude (PINTO Jr. e PINTO, 2000). Contudo, uma intensa instabilidade atmosférica pode originar um rápido movimento convectivo com grande expansão vertical de uma massa de ar úmida, e geralmente apresenta precipitação, granizo e descargas elétricas.

Segundo Gomes (2003), a existência de cargas elétricas em nuvens é gerada pelas colisões de diferentes partículas de gelo no interior da nuvem de tempestade, que transferem cargas entre as partículas pelo processo indutivo e termoelétrico, entretanto muito pouco se sabe sobre os detalhes dos processos de colisões das partículas.

Também ainda não se sabe exatamente como as nuvens de tempestades se tornam carregadas, pois a estrutura elétrica de uma nuvem de tempestade é muito complexa. Esta estrutura elétrica ocorre devido aos processos macrofísicos, que atuam em escalas de quilômetros, e aos processos microfísicos, que atuam em escala de milímetros, ocorrendo simultaneamente dentro da nuvem. Assim, para entender a estrutura elétrica da nuvem é fundamental identificar estes diversos processos para determinar a importância 
relativa de cada um. O resultado destes são cargas intensas produzidas no interior da nuvem com valores que podem variar de algumas poucas dezenas até poucas centenas de coulombs. São estas cargas que originam os relâmpagos (GOMES, 2003).

Contudo, sabe-se que as condições meteorológicas em escala sinótica, o relevo e os centros urbanos podem exercer influência sobre a frequência das descargas elétricas, sua polaridade, porcentagem e a intensidade de relâmpagos. Nos grandes centros urbanos, os efeitos das ilhas de calor e da poluição, "aparentemente alteram a distribuição das cargas das nuvens de tempestades, afetando a densidade e a polaridade dos relâmpagos" (GOMES, 2003; NACCARATO, 2006).

\section{CENTROS URBANOS E AS DESCARGAS ELÉTRICAS ATMOSFÉRICAS}

Analisar os efeitos dos centros urbanos sobre a ocorrência de raios é complexo, pois ainda não são totalmente esclarecidos os processos desta relação.

Atualmente, algumas pesquisas relacionam a influência de áreas urbanas, com enfoque nas ilhas de calor, na formação das nuvens e/ou da precipitação (e.g.: CHANGNON, 1980; CHANGNON et al., 1981. PEREIRA FILHO et al., 2004; GOMES, 2003; NACCARATO, 2006).

Embora diversos trabalhos ressaltem fortes evidências da influência das áreas urbanas na atividade das tempestades, os possíveis mecanismos físicos associados a essas variações ainda não foram totalmente comprovados (e. g.: WESTCOTT, 1995; SORIANO e PABLO, 2002; ORVILLE et al., 2001; NACCARATO et al., 2003; PINTO et al., 2004).

Segundo Gomes (2003) e Naccarato (2006), várias pesquisas tem comprovado que os grandes centros urbanos afetam a ocorrência de descargas elétricas na atmosfera. Os autores concluíram que existe uma possível relação entre a distribuição espacial da atividade de raios, a poluição atmosférica (material particulado), o relevo e os sistemas atmosféricos.

Gomes (2003) observou através de técnicas estatísticas que os parâmetros característicos dos relâmpagos nos centros urbanos são significativamente maiores que nas regiões circunvizinhas.

Naccarato (2006, p.306) ao analisar as características dos relâmpagos na região sudeste do Brasil, no período entre 1999 a 2004, concluiu que aparentemente, a poluição dos grandes centros urbanos interfere na frequência deste fenômeno, "quando a poluição atmosférica atinge um determinado nível crítico, ela passa a modular a convecção através da alteração dos perfis de temperatura na camada limite".

Em sua pesquisa observou a densidade de raios no Estado de São Paulo, entre 1999 a 2004, e constatou uma grande densidade na área metropolitana de São Paulo, Vale do Rio Paraíba especialmente no município de São José dos Campos e na área do município de Campinas no interior do estado. Entretanto, a densidade diminui gradualmente em direção ao interior (NACCARATO, 2006). 
Para Naccarato (2006, p. 279-280), existem duas possíveis hipóteses para explicar este fenômeno:

1) Hipótese do Aerossol: propõe que a poluição urbana eleva a concentração de núcleos de condensação (NC) na atmosfera, provocando alterações nos processos microfísicos das nuvens de tempestades. Isso, por sua vez, afeta os mecanismos de separação de cargas, os quais dependem da concentração, fase e tamanho das partículas dentro da nuvem. Com isso haveria um aumento da atividade elétrica e variações na proporção entre as polaridades;

2) Hipótese Termal: propõe que as ilhas de calor urbanas são responsáveis pela intensificação da convecção local (diretamente relacionado com o aumento das velocidades de ascensão do ar potencializando assim a eletrificação das nuvens de tempestades sobre as cidades). Isso promove então um aumento na atividade de raios.

É importante ressaltar que nos grandes centros urbanos, com maior complexidade na relação (alteração) com o ambiente natural, geralmente encontram-se altos índices de número/densidade de descargas elétricas, sendo comumente acentuados quando comparados com as regiões periféricas, ou áreas urbanas menores. Porém, destaca-se que existem inúmeros fatores que influenciam no desenvolvimento deste fenômeno, como algumas atividades rurais, por exemplo, portanto nem sempre esta relação se faz presente.

Em seu estudo, Naccarato (2006) observou que a região de Campinas e a região de São José dos Campos apresentaram um aumento de 50 a $60 \%$ no total de raios em comparação com as cidades circunvizinhas. Para a Região Metropolitana de São Paulo (RMSP), o aumento é ainda mais expressivo, atingindo de 150 a $200 \%$.

Naccarato (2006) realizou ainda "uma comparação entre a temperatura aparente da superfície (obtida através da imagem da banda termal do satélite LandSat-7), que caracteriza a ilha de calor urbana[...]", a distribuição geográfica dos raios na RMSP e um mapa com a área urbana do município de São Paulo (Figura 1). 


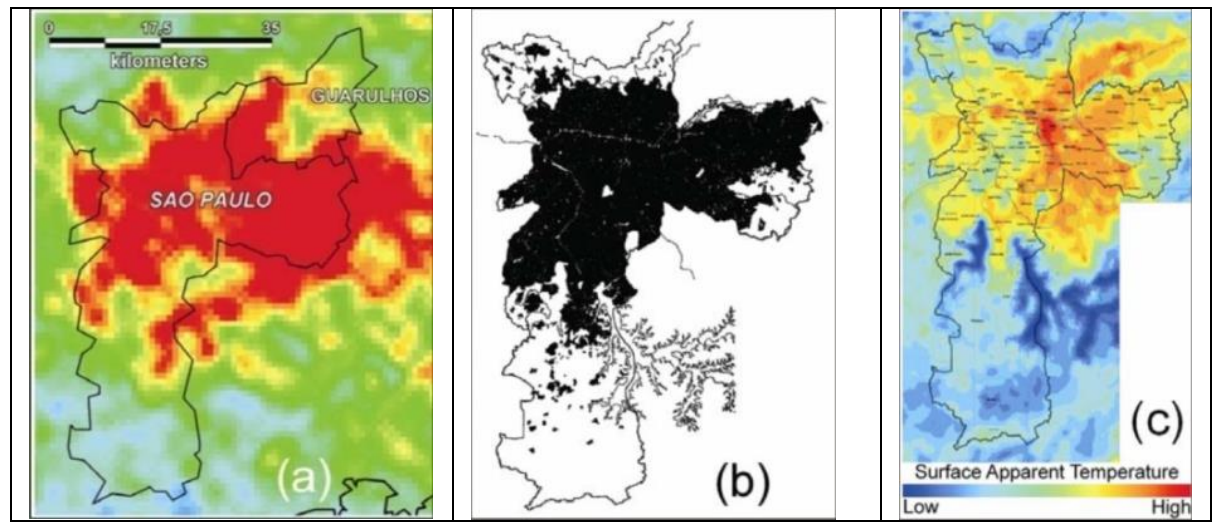

Figura 1: (a) Densidade de raios na RMSP $(1 \times 1 \mathrm{~km})$. (b) Localização da área urbana do município de São Paulo (em preto). (c) Temperatura aparente da superfície obtida a partir da imagem da banda termal do satélite LandSat-7, caracterizando a ilha de calor urbana.

Fonte: Naccarato (2006, p. 277).

O autor concluiu que os relâmpagos se concentram sobre a área urbanizada, onde ocorrem as maiores temperaturas, estreitando a correlação espacial entre os três fatores.

As questões apontadas acima fazem referência às influências exercidas no microclima urbano, que nem sempre são apresentadas, como demonstrado adiante.

\section{PROCEDIMENTO METOdOLÓGICO}

Os estudos sobre descargas elétricas atmosféricas são recentes, especialmente no Brasil. Sendo assim, praticamente não existem pesquisas com enfoque geográfico sobre tais eventos, portanto foi imprescindível a realização do levantamento bibliográfico sobre os raios em diversas áreas do conhecimento científico. Apesar da dificuldade em transpor a linguagem meteorológica para a geográfica, a bibliografia encontrada muito esclareceu sobre as descargas elétricas, suas ocorrências nas tempestades severas e seus impactos.

Para as análises e espacialização do número e densidade de raios para todos os municípios do Estado de São Paulo no período de 2005 - 2006 foi utilizado o ranking desenvolvido pelo ELAT (Grupo de Eletricidade Atmosférica) do INPE (Instituto Nacional de Pesquisas Espaciais).

Já para espacialização da frequência de raios e mortes/feridos decorrentes dos mesmos, foi utilizado o banco de dados de eventos severos elaborado pelo IPMET/UNESP - Campus de Bauru, a partir de informações coletadas sobre todos os municípios paulistas junto à Coordenadoria Estadual de Defesa Civil/SP de 2000 a 2007, contendo os danos ocorridos, os fenômenos atmosféricos que os propiciaram, localização, data e hora dos sinistros.

Para espacializar as informações referentes aos raios foi utilizada a base espacial de informações municipais do Estado de São Paulo, no formato Ano 9 - Vol. 13 - JUL/DEZ 2013 
shapefile do Sistema de Informações Georreferenciadas EstatCart, desenvolvido pelo Instituto Brasileiro de Geografia e Estatística - IBGE (BRASIL, 2004), e que foi empregada, com sucesso, como plano de informação espacial em André et al. (2008a) e André et al. (2008b).

O Sistema de Informações Georreferenciadas EstatCart, que foi desenvolvido pelo IBGE, possibilita associar uma visão espacial a uma base de informações. Criado para atender à crescente procura de informações estatísticas e geográficas e/ou em escala dos setores censitários, o sistema possibilita o acesso a base de informações municipais e bases do Censo como universo, amostra, setor censitário e área de ponderação. Com o EstatCart é possível criar mapas temáticos, calcular indicadores e tabelas que podem ser impressas ou exportadas. A informação também pode ser analisada na sua dimensão espacial, o que torna a análise imediata (ANDRÉ et al., 2008a).

Esta base de dados foi exportada para o Sistema de Informação Geográfica ArcView (ESRI, 1996) e os dados sobre os raios, mortes e/ou feridos foram inseridos.

O SIG ArcView possui um formato de armazenamento de dados vetoriais baseados em arquivos, isto é, os atributos dos elementos geográficos são armazenados em um banco de dados denominado de tabela de atributos. Cada linha desta tabela contém as informações descritivas de uma única feição e as colunas ou campos definidos na tabela são as mesmas para cada linha (CÂMARA E MONTEIRO, 2004).

A ligação entre as feições geográficas e a tabela de atributos é garantida pelo modelo geo-relacional, isto é, um identificador único efetua a ligação entre ambos, mantendo uma correspondência entre o registro espacial e o registro de atributos. Segundo Câmara e Monteiro (2004), uma vez que esta conexão é estabelecida, podem-se apresentar informações descritivas sobre o mapa e armazenar outras.

Nesta fase do trabalho, a tabela de atributos foi alimentada com os dados, e o resultado final deste procedimento são os mapas temáticos. Estes mapas identificaram a localização dos municípios atingidos por raios, à densidade ou vítimas fatais e/ou feridos (isolados ou associados) registradas pela Defesa Civil, no Estado de São Paulo.

Para as análises estatísticas dos dados de descargas elétricas atmosféricas do ELAT/INPE (2005 e 2006) foram realizados o coeficiente de incidência e o coeficiente de letalidade ou fatalidade.

O coeficiente de incidência (LAURENTI et al., 1987) é o risco de um indivíduo da população vir a adquirir uma doença, ou no caso deste trabalho, de ser atingindo por uma descarga elétrica atmosférica. Este coeficiente é dado pela relação: 


\title{
número de pessoas atingidas por descargas elétricas atmosféricas
}

total da população na área

O coeficiente de letalidade ou fatalidade representa o risco de um indivíduo morrer na população que apresenta determinada doença. Neste caso seria o risco de morte na população exposta a um evento de descargas elétricas atmosféricas (LAURENTI et al., 1987). Assim, o cálculo é expresso por:

\author{
número de óbitos por descargas elétricas atmosféricas \\ número ou densidade de descargas elétricas atmosféricas na área

\section{RESULTADOS}

A configuração do relevo é um fator importante para descrever os fenômenos climáticos no Estado de São Paulo, sendo composto por faixa litorânea, planalto atlântico, planalto ocidental e depressão periférica. O planalto atlântico possui forte relevância, principalmente pluviométrica, uma vez que este barra os ventos úmidos de origem oceânica, resultando nas denominadas "chuvas orográficas". Nimer (1979) afirma que "[...] a orografia determina uma séria de variedades climáticas, tanto no que se refere à temperatura quanto à precipitação".

Monteiro (1976) descreve o clima do estado como tendo "[...] caráter mesotérmico, forte amplitude térmica e farta distribuição anual de chuvas".

Sua localização geográfica Ihe confere características climáticas complexas, pois seu território apresenta condições para o choque entre as massas de ar tropicais (tropical atlântica, equatorial continental e tropical continental) e polares (polar atlântica). "Segundo Monteiro (1973), as três massas atingem o Brasil meridional através de três grandes correntes de perturbação: as de estenordeste, as de noroeste e as de sul" (MAIA, 2007).

Logo, o Estado de São Paulo situa-se no limite de duas zonas climáticas intertropical e subtropical. A primeira sofre influência das massas equatoriais e tropicais, sendo que, a segunda apresenta influência de massas tropicais e polares.

A primeira grande corrente de perturbação resulta da atuação da Massa Tropical Atlântica (mTa), influenciando a região durante os doze meses do ano, porém, sua ação no inverno é atenuada.

A segunda é formada pela influência de duas massas: a Massa Equatorial Continental (mEc) e a Massa Tropical Continental (mTc). Sua ação resulta nas denominadas chuvas convectivas de oeste, também conhecidas popularmente 
por "chuvas de verão". Estas são de curta duração, porém intensas. Nimer (1979) observa que "[...] ao contrário das chuvas frontais (provocadas pela ação direta das frentes polares) que costumam ser intermitentes durante dois, três, ou mais dias, as chuvas de verão (chuvas de convergência) duram poucos minutos".

A terceira e última corrente de perturbação tem sua origem na Massa Polar Atlântica, sendo que estas "são as responsáveis pela atuação da Frente Polar Atlântica (FPA)" (MAIA, 2007). Esta tem sua atuação diferenciada de acordo com a sazonalidade. No inverno, estas resultam em precipitações pouco expressivas, sendo os principais fatores, a pouca umidade encontrada no ar quente da Massa Tropical Marítima em ascensão e a falta de umidade do Anticiclone Polar, devido ao trajeto continental com características secas resultantes do inverno. Já no verão, a corrente supracitada torna-se a principal responsável pela precipitação no estado.

Nimer (1979) explica que "ao atingir a região sudeste, a FP não possui, na maioria das vezes, energia suficiente para mantê-la em constante FG (frontogênese, isto é, avanço), estabelecendo-se daí o equilíbrio dinâmico entre a alta do Atlântico Sul e a Alta Polar". Assim, esta permanece semi-estacionária sobre a região, podendo se dissipar em poucos dias, ou continuar atuante por mais de 10 dias, até finalmente se dissipar.

Monteiro (2000) examinou a influência das principais correntes de circulação atmosférica da vertente atlântica da América do Sul e concluiu que a Frente Polar Atlântica "é a principal responsável pela gênese das chuvas" (MONTEIRO, 2000). A participação desta no clima do sudeste brasileiro é geralmente responsável pela maior parte da precipitação em todas as estações. Outro fator que aumenta a importância desta no regime pluviométrico do estado é que a "sua mobilidade e dinamismo próprios são os reguladores da participação dos sistemas intertropicais".

Esta observação é relevante quanto ao perfil pluviométrico do estado. Segundo Monteiro (2000),

A atuação percentual das massas de ar, base mesma da análise rítmica, serviu de fundamento ao traçado do limite das duas unidades climáticas regionais. Partindo daí e baseados nas variações do ritmo e distribuição quantitativa espacial dos elementos disponíveis dentro do quadro morfológico, chegamos a nove sub-unidades. Estas, subdividindo-se ainda segundo fácies mais nitidamente observáveis chegam ainda a exibir dezessete feições climáticas.

Assim, a região sul do estado apresenta maior índice pluviométrico, sofrendo maior influência da Frente Polar Atlântica; e a região norte, com exceção do litoral e do Planalto Atlântico Norte, apresenta menor umidade. O litoral e o Planalto Atlântico Norte possuem forte influência orográfica exercida em relação à massa Tropical Atlântica (MONTEIRO, 2000). 
Quanto ao fator morfológico, citado no início, além do que já foi exposto, podese acrescentar sinteticamente o seguinte parágrafo:

A natureza predominantemente planáltica do Estado de São Paulo confere-Ihe um caráter de mesotermia subtropical, que, mesmo sujeito a temperaturas elevadas no verão, beneficia-se de ondas de refrigério graças à participação dos sistemas atmosféricos extratropicais [...] (Monteiro, 1976).

A expressiva urbanização pode ainda exercer influência no clima do estado, pois enorme concentração de construções e poluentes lançados ao ar constantemente, agem diretamente no microclima, podendo desta forma, alterar a complexa dinâmica do macroclima (MONTEIRO, 2000).

Os fatores apontados acima, resumidamente, caracterizam o clima do Estado de São Paulo, como sendo tipicamente tropical, com altas temperaturas e altos índices pluviométricos no verão, abrangendo a maior parte da primavera; e no inverno, e maior parte do outono; temperaturas mais amenas e uma drástica redução na umidade, com pequenos períodos de estiagem em alguns pontos.

Ao comparar a classificação climática proposta por Monteiro (2000) e o número de raios no Estado de São Paulo, nota-se semelhanças (Figura 2). 


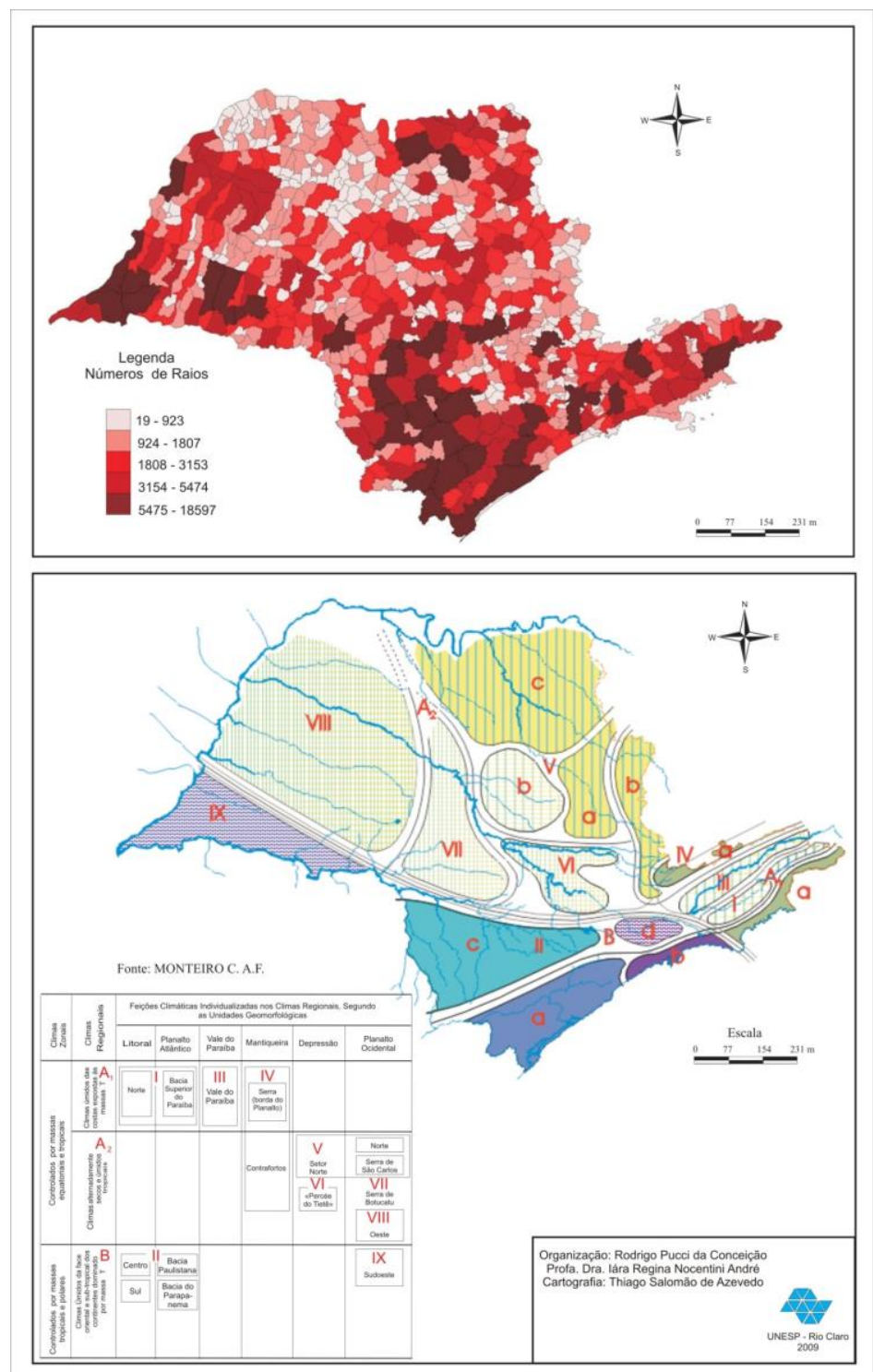

Figura 2: a) número de descargas elétricas atmosféricas b) classificação climática do Estado de São Paulo,

Fonte: Monteiro (2000) e Brasil (2008)

A maior quantidade de raios por município concentra-se na faixa litorânea e na parte sul do estado, principalmente na região do Vale do Ribeira e na Serra do Paranapiacaba, onde encontram-se maior aglomerado de municípios com altos índices de número de raios. A região do Pontal do Paranapanema também se destaca na grande concentração de número de raios. No norte do estado, observa-se também uma grande quantidade de raios na região de Ribeirão Preto. Segundo Monteiro (2000), esta região "mantém a nitidez do período seco, habitualmente entre 100 e 200 mm de chuvas e apenas 10 a 15 dias de precipitação [...] contrastando com um período chuvoso de maiores índices [...]" que o das regiões circundantes. Talvez, este fato explique o grande número de relâmpagos apresentado na região, uma vez que este estudo analisa apenas 2005-2006, não diferenciando as características sazonais. 
Monteiro (2000) ressalta que no Estado de São Paulo,

A frouxidão da faixa de limites deixa ver áreas de estação seca na unidade meridional embora o caráter básico da mesma seja de farta distribuição pluvial no decorrer do ano. Do mesmo modo, na unidade setentrional a influência de combinações de fatores geográficos, notadamente o relevo, responde pela ocorrência de áreas permanentemente pluviosas em meio a uma predominância de nitidez de períodos secos (Monteiro, 2000).

Contudo, é necessário salientar que a gênese das frentes podem gerar tempestades severas que desenvolvem descargas elétricas, portanto as áreas que apresentaram maiores números de raio no período, possivelmente estiveram mais ativamente sobre a influência das frentes. Entretanto, é importante enfatizar que os dados na elaboração do mapa temático sobre o número de raios referem-se somente para 2005-2006 e segundo Monteiro (1976) "embora ocorram modificações sensíveis nas quantidades de chuvas caídas em diferentes anos à gênese das chuvas permanece relativamente à mesma".

Monteiro (2000) expõe ainda a possível influência da frequente passagem de sistemas frontais no litoral de São Paulo, induzindo a formação de tempestades convectivas, resultando na grande incidência de raios observada em praticamente toda faixa litorânea do estado. Este fator pode atuar como um dos elementos colaboradores na geração de tempestades elétricas, somando-se a outras questões, como temperatura, umidade e poluição.

A espacialização da densidade de descargas elétricas atmosféricas para 20052006 elaborado com os dados divulgados pelo ELAT/INPE (Figura 3) é similar aos encontrados por Naccarato (2006), logo se deve considerar o possível fator de urbanização. Fica subentendido que as maiores densidades de descargas elétricas estão relacionadas com os municípios com maior urbanização. Assim, de acordo com as semelhanças encontradas entre 2005-2006 e os anos anteriores observados por Naccarato (2006), os resultados demonstraram fortes indícios de que as ilhas de calor e a poluição influenciam no aumento da densidade de descargas elétricas principalmente nos aglomerados urbanos. 


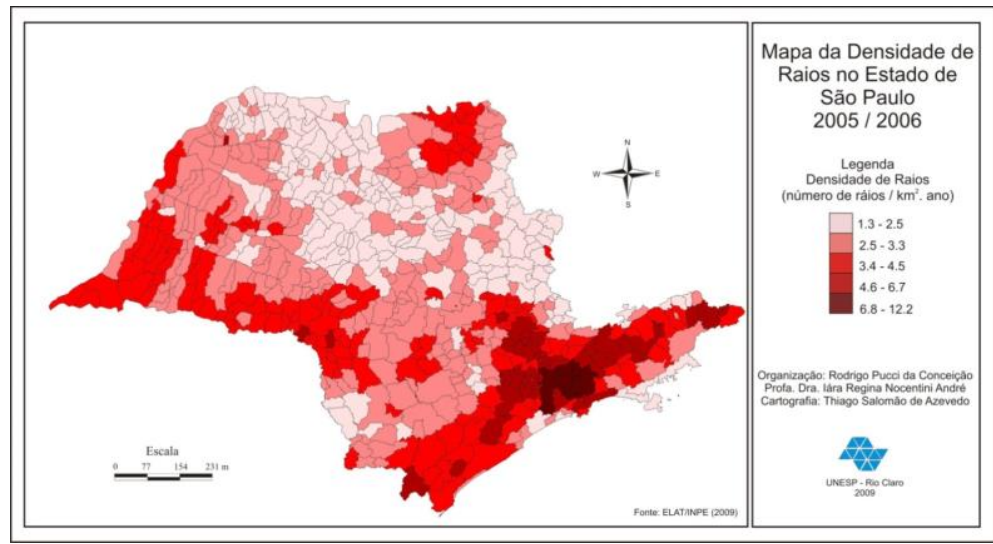

Figura 3: Densidade de descargas elétricas atmosféricas no Estado de São Paulo 2005-2006

Contudo, ao relacionar o número (Figura 2a) e a densidade (Figura 3), tem-se uma observação interessante nos municípios de Piracicaba e Sorocaba. Apesar de Piracicaba apresentar área municipal maior do que o município de Sorocaba, o índice de urbanização do segundo município é maior que o primeiro. Mesmo assim, o município de Piracicaba apresentou nos anos de 2005/2006, maior número e densidade de raios que Sorocaba. Em Piracicaba foram registrados 6539 raios e densidade igual a 3,183 raios $/ \mathrm{km}^{2}$.ano, já em Sorocaba registrouse 1879 raios e densidade igual a 2,789 raios/km2.ano. Uma característica aceitável para explicar está diferença de descargas elétricas atmosféricas no município de Piracicaba, seria a prática de queimada da cana-de-açúcar, muito comum na região, tornando-se foco de acúmulo de material particulado, somando-se as atividades urbanas, como citado no parágrafo anterior.

\section{DESCARGAS ELÉTRICAS ATMOSFÉRICAS E VÍTIMAS}

O Estado de São Paulo possui a maior população do país com cerca de 40 milhões de habitantes distribuídos em 645 municípios. É considerado o "motor econômico" do Brasil, pois é responsável por mais de $31 \%$ do PIB nacional. Assim, é considerado o estado mais rico da União, apresenta-se com alto Índice de Desenvolvimento Humano (IDH) e urbanização (SÃO PAULO, 2009).

Como visto anteriormente, São Paulo está diretamente sobre a influência das frentes e consequentemente das tempestades severas e descargas elétricas atmosféricas. Com uma área densamente povoada é natural que inúmeros casos de vítimas (fatais e/ou feridos) ocorram no decorrer dos anos.

Segundo a Universidade Federal Rural do Rio de Janeiro - UFRRJ (2009), o Brasil registrou cerca de 100 milhões de raios apenas no ano 2000 e 100 pessoas morreram em função destas descargas elétricas atmosféricas. Estes fenômenos causam também prejuízos anuais em torno de US\$200 milhões ao país e danificam as linhas de transmissão de energia, de telefonia, as indústrias e causam incêndios florestais.

Para a UFRRJ (2009), a probabilidade de uma pessoa ser atingida por um raio é muito pequena, segundo cálculos estatísticos, cerca de uma para um milhão e a chance de sobreviver a uma descarga direta é de apenas $2 \%$. Em $30 \%$ dos 
casos de pessoas atingidas por descargas elétricas atmosféricas, incluindo as indiretamente atingidas, as vítimas apresentam óbito por parada cardíaca ou respiratória. Já os $70 \%$ restantes apresentam sequelas, como perda de memória e diminuição da capacidade de concentração. Quando atingem uma pessoa, as descargas elétricas atmosféricas causam enormes danos ao coração, pulmões, sistema nervoso central, além de graves queimaduras decorrentes do aquecimento e de várias reações eletroquímicas.

Foram elaborados alguns mapas temáticos com base nos registros da Defesa Civil do Estado de São Paulo para vítimas fatais (Figura 4) e/ou feridos (Figura 5), em 2005 a 2006. Ao relacionar a ocorrência de descargas elétricas atmosféricas nota-se baixa correlação com a densidade e número de raios ocorridos (registrados) nos municípios paulistas.

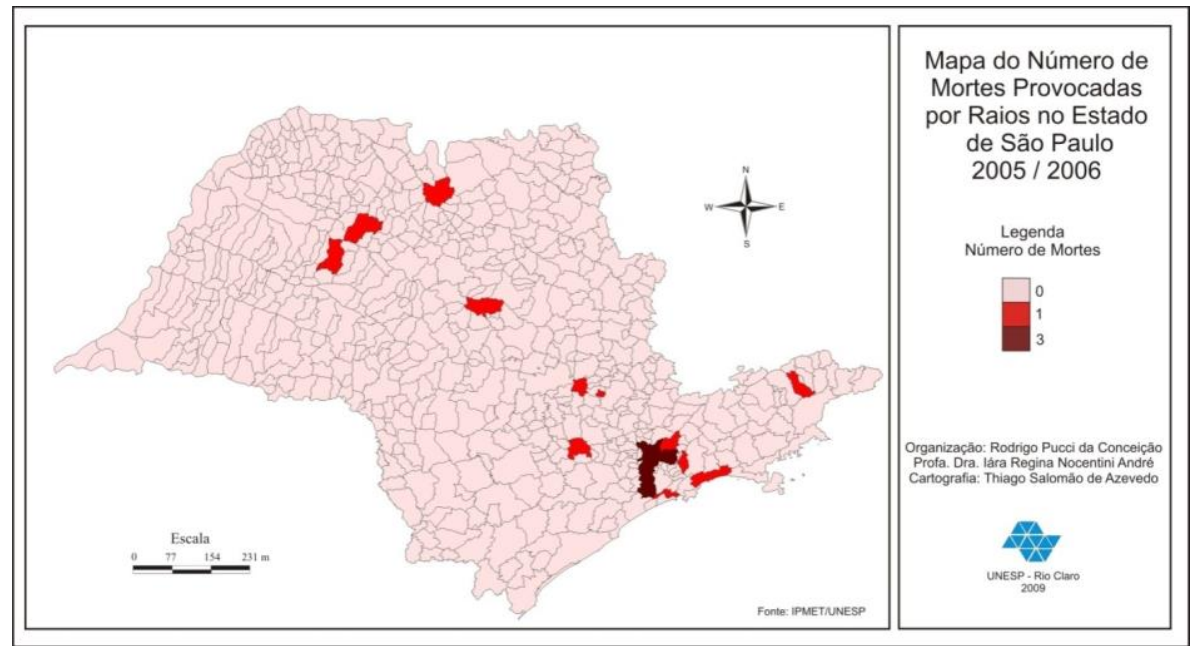

Figura 4: Vitimas fatais decorrentes de descargas elétricas atmosféricas Fonte: dados IPMET (2007)

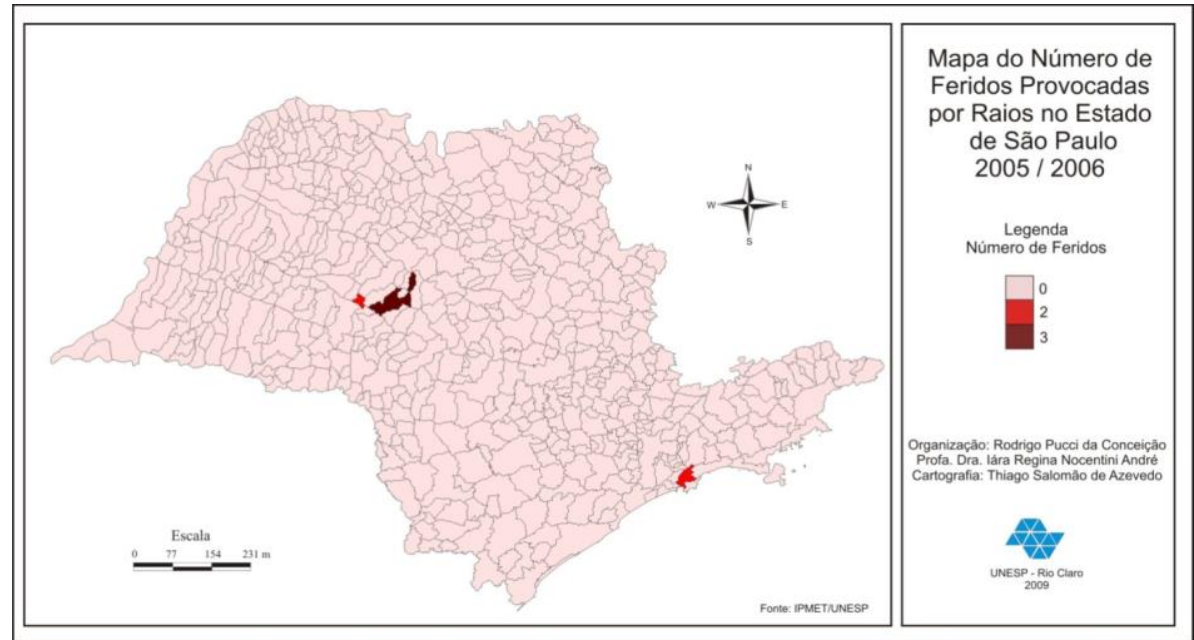

Figura 5: Feridos decorrentes de descargas elétricas atmosféricas Fonte: dados IPMET (2007) 
Para a elaboração dos coeficientes de incidência e letalidade foram utilizados os dados divulgados pelo ELAT/INPE dos municípios do Estado de São Paulo que apresentaram vítimas decorrentes de raios (Tabela 1) no período de 2005/2006.

Tabela 1

Municípios que apresentaram vítimas decorrentes de descargas elétricas atmosféricas em 2005/2006.

\begin{tabular}{|c|c|c|c|c|c|}
\hline Localidade & Número de Raios & População 2006 & Densidade Raio & Vítimas & Mortes \\
\hline Hortolândia & 421 & 185840 & 6,77 & 1 & 1 \\
\hline São Vicente & 574 & 322535 & 3,87 & 1 & 1 \\
\hline Santa Bárbara d'Oeste & 1722 & 182962 & 6,34 & 1 & 1 \\
\hline Sorocaba & 1879 & 564763 & 4,18 & 1 & 1 \\
\hline Boa Esperança do Sul & 2033 & 13669 & 2,94 & 1 & 1 \\
\hline Lorena & 2526 & 82297 & 6,10 & 1 & 1 \\
\hline Olímpia & 2732 & 48336 & 3,40 & 1 & 1 \\
\hline Penápolis & 2770 & 56764 & 3,91 & 1 & 1 \\
\hline Suzano & 2863 & 274727 & 13,91 & 1 & 1 \\
\hline José Bonifácio & 2930 & 31027 & 3,41 & 1 & 1 \\
\hline Bertioga & 3727 & 39666 & 7,58 & 1 & 1 \\
\hline Guarulhos & 4089 & 1247109 & 12,86 & 1 & 1 \\
\hline São Paulo & 18597 & 10824242 & 12,21 & 3 & 3 \\
\hline Santos & 1964 & 428370 & 7,01 & 2 & 0 \\
\hline
\end{tabular}




\begin{tabular}{|c|c|c|c|c|c|}
\hline Pirajuí & 2383 & 21535 & 2,91 & 3 & 0 \\
\hline Júlio de Mesquita & 345 & 4435 & 1,79 & 2 & 0 \\
\hline
\end{tabular}

Fonte: ELAT/INPE (2008)

O coeficiente de incidência, ou seja, o risco de um indivíduo da população ser atingido por uma descarga elétrica, no período de 2005/2006, está representado na figura 6 .

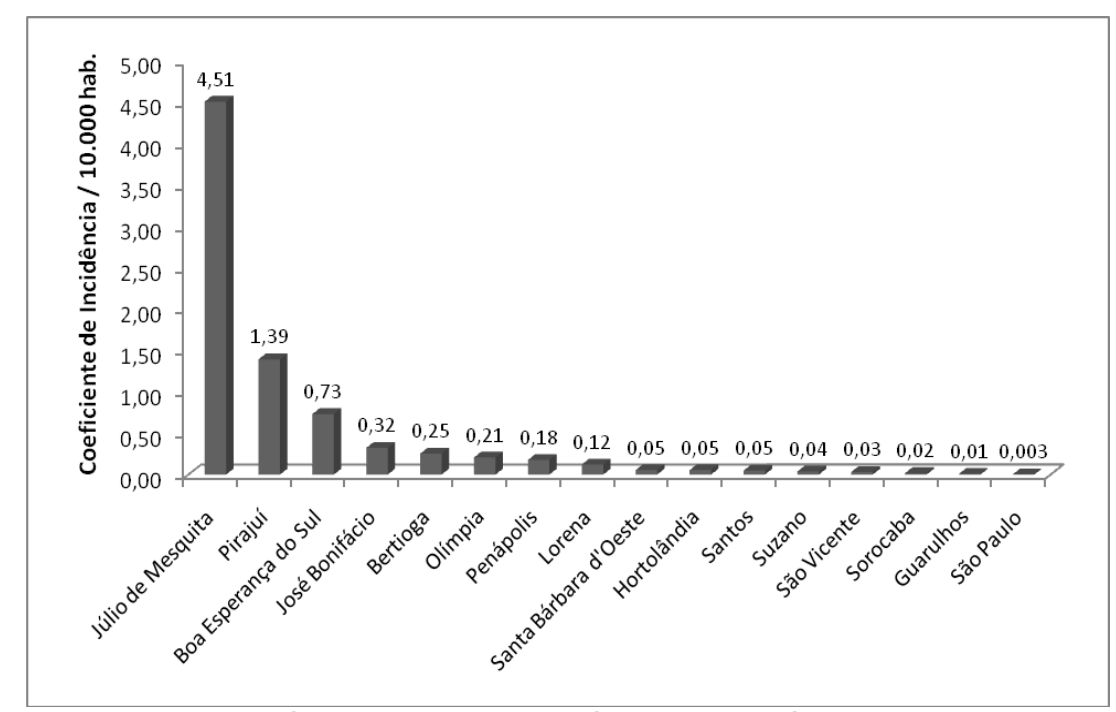

Figura 6: Coeficiente de incidência de descargas elétricas atmosféricas por 10.000 hab.

Segundo o resultado do cálculo deste coeficiente, o município de Julio de Mesquita apresentou o maior coeficiente. Este fato ocorre porque este município, apesar de ter registrado baixa frequência de descargas elétricas no período e possuir baixa concentração populacional, apresentou duas vítimas deste fenômeno atmosférico. Neste caso, a quantidade de vítimas é muito representativa justamente por considerar o número de descargas elétricas e a concentração populacional do município.

Inversamente ocorre no município de São Paulo, que apesar de apresentar maior número de vítimas, possui maior concentração populacional e registrou maior número de descargas elétricas atmosféricas.

O coeficiente de letalidade, que representa o risco de morte na população exposta a um evento de descargas elétricas atmosféricas, foi calculado para o número e densidade de raios. 


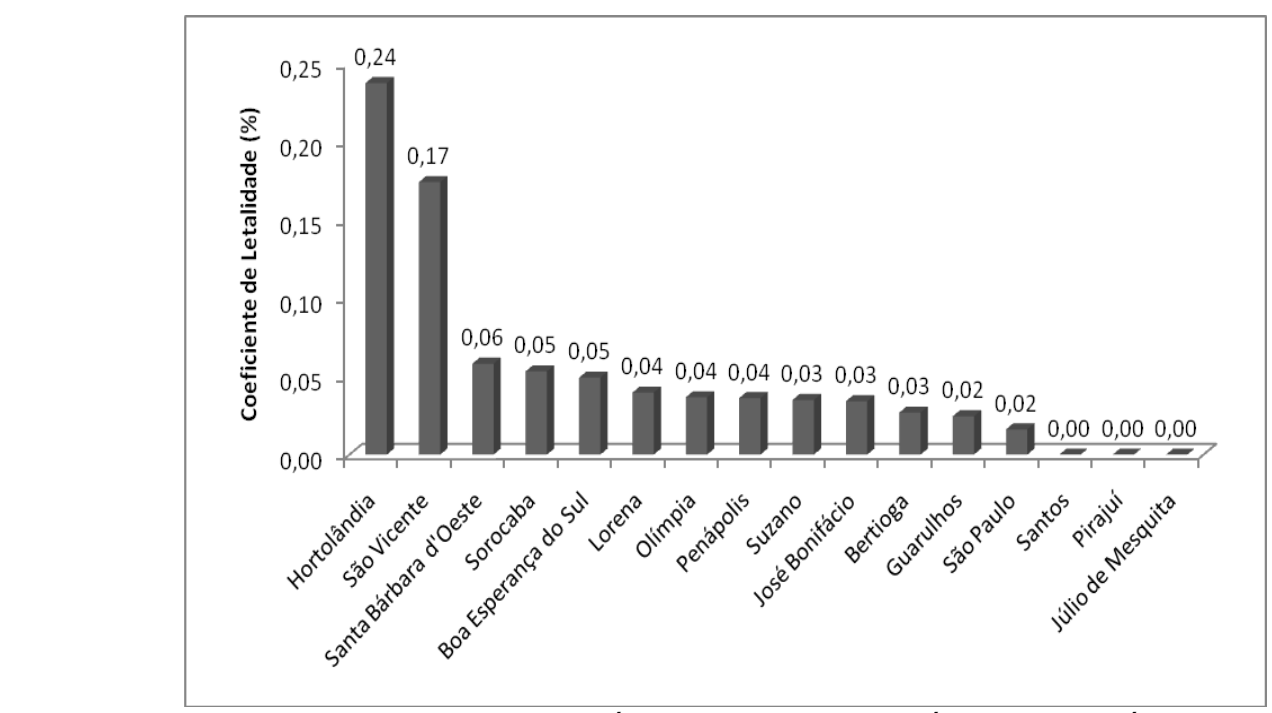

Figura 7: Coeficiente de letalidade pelo número de descargas elétricas atmosféricas

De acordo com a figura 7, observa-se que o coeficiente de letalidade, quando considerado o número de descargas elétricas atmosféricas, é baixo, contrariando as estatísticas mundiais apresentadas pela Cruz Vermelha (NACCARATO, 2006). Contudo, deve-se ressaltar que para o cálculo deste coeficiente, foram analisados apenas os anos de 2005/2006 no Estado de São Paulo.

Apesar do baixo índice, os municípios de Hortolândia e São Vicente apresentaram coeficientes expressivamente maiores, quando comparados aos outros municípios. Isto ocorre, porque apesar do número de óbitos ser igual na maioria dos municípios, o número de raios naqueles, foi extremamente inferior. Os demais municípios apresentaram coeficientes aproximados, que variam entre $0,06 \%-0,02 \%$.

Já o município de São Paulo, que obteve coeficiente muito baixo, registraram-se três óbitos, contudo, o número de descargas elétricas atmosféricas foi muito maior que o restante dos municípios.

Já ao analisar o coeficiente de letalidade pela densidade de descargas elétricas atmosféricas (Figura 8), o resultado é muito mais expressivo. O município de Boa Esperança do Sul, por exemplo, ultrapassa os $30 \%$ e o município de Suzano, cujo coeficiente é de $7,19 \%$, é o que menos oferece risco. 

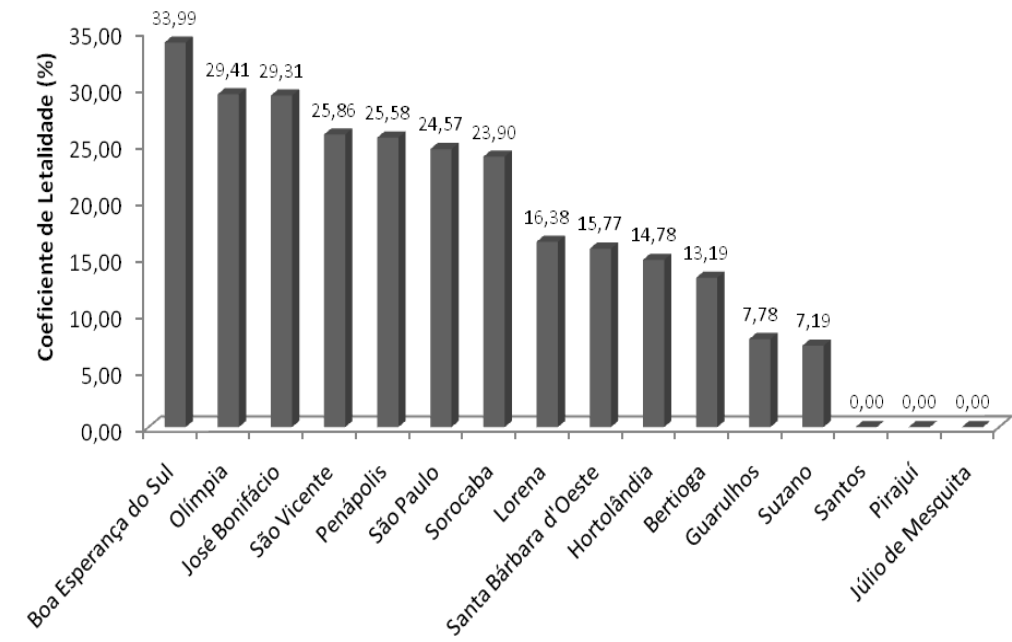

Figura 8: Coeficiente de letalidade pela densidade de descargas elétricas atmosféricas

O exposto acima se deve principalmente pelas variantes área e número de raios, que definiram a densidade, principal fator diferencial neste caso. Isto fica claro quando se observa os municípios de Suzano e Guarulhos, que apresentam menor área, e número de descargas elétricas relativamente elevado no ano em questão. O oposto pode ser observado nos municípios de Boa Esperança do Sul e Olímpia. Já no caso de São Paulo, a área do município é grande, o número de descargas elétricas atmosféricas registrado no mesmo período, também é grande, e o número de óbitos resultante (Tabela 1 ) foi superior a todos os outros municípios, o que deixou o município numa situação intermediária.

Os três municípios que apresentaram porcentagem igual a $0 \%$, nos casos das figuras 7 e 8 , se deve a falta de óbitos (Tabela 1), sendo que o número de vítimas assinalado faz referência aos feridos atingidos por raios.

\section{CONCLUSÃO}

Para o desenvolvimento deste trabalho, foi fundamental a realização de um levantamento bibliográfico ligado a outras áreas do conhecimento científico, para o entendimento do fenômeno atmosférico abordado. Porém, os termos técnicos utilizados, dificultaram a adaptação na linguagem geográfica nas características ligadas aos fenômenos e suas relações com o meio geográfico.

O período estudado foi muito curto (2005/2006) para se fazer qualquer afirmativa. Isto mostra a necessidade de um aprimoramento para desvendar todos os processos, riscos e impactos destes eventos atmosféricos. Assim, um estudo com maior escala temporal possivelmente revelará o aspecto geográfico das descargas elétricas atmosféricas. 
É fundamental o trabalho em conjunto, entre autoridades, comunidade científica, e a população, para diminuir os danos gerados pelos eventos climáticos extremos, dentre eles, as descargas elétricas atmosféricas.

\section{REFERÊNCIAS BIBLIOGRÁFICAS}

ANDRÉ, I. R. N. Algumas considerações sobre mudanças climáticas e eventos atmosféricos severos no Brasil. Climatologia e Estudos da Paisagem, Rio Claro, v.1, n. 1/2, p.1-9, jul./dez, 2008a.

ANDRE, I. R. N.; TAVARES, A. C.; FERREIRA, N. J.; AZEVEDO, T. S.; MUTTI, R. G. Proposta metodológica para espacialização de ocorrências registradas na Defesa Civil em decorrência de tempestades. In: Congresso Brasileiro de Meteorologia, 15, 2008b, Aracaju, Brasil. (no prelo).

BRASIL. Instituto Brasileiro de Geografia e Estatística - IBGE. Sistema de informações georreferenciadas Estatcart. Rio de Janeiro, 2004 CD-ROM.

BRASIL. Instituto Nacional de Pesquisas Espaciais. Grupo de Eletricidade Atmosférica (INPE-ELAT). Descargas Atmosféricas. Disponível em: <http://www.inpe.br/webelat/homepage/>. Acesso em: 10 out. 2008.

CÂMARA, G.; MONTEIRO, A. M. U. Conceitos básicos em ciência da geoinformação. In: CÂMARA, J. et al. (Ed.). Introdução a ciência da geoinformação. São José dos Campos: INPE, 2004. Disponível em: <http://www.dpi.inpe.br/gilberto/livros.html>. Acesso em: mar. 2008.

CHANGNON, S. A. Evidence of urban and lake influences on precipitation in the Chicago area. Journal of Applied Meteorology, v. 19, n. 10, p. 1137-1159, 1980 .

CHANGNON, S. A. et al. METROMEX: a review and summary. Boston: American Meteorological Society, 1981. $181 \mathrm{p}$.

ESRI. Using ArcView GIS. Redlands: Esri, 1996, 350 p.

FORSDYKe, A. G. Previsão do tempo e clima. São Paulo: Melhoramentos-EDUSP, 1975. (Série Prisma - o conhecimento em cores, v. 17).

GOMES, M. A. S. S. Estudo dos relâmpagos na Região Sudeste do Brasil em função das características geográficas. 2003. 149 p. Tese (Doutorado em Geofísica Espacial) - Instituto Nacional de Pesquisas Espaciais, São José dos Campos, 2003.

LAURENTI, R. et al.; Estatísticas de saúde. São Paulo, E.P.U., 2a Ed., 1987.

LIMA, K. C. Descargas elétricas atmosféricas em sistemas convectivos de mesoescala no sul da América do Sul. 2005. 118 p. Dissertação (Mestrado) - Programa de Pós-Graduação em Meteorologia. Faculdade de Meteorologia. Universidade Federal de Pelotas, Pelotas, 2005.

MAIA, D. C. Impactos pluviais na área urbana de Ribeirão Preto-SP. 2007. 153 p. Tese (Doutorado) - Instituto de Geociências e Ciências Exatas. Universidades Estadual Paulista, Rio Claro, 2007.

MARCELINO, I. P. V. O.; Análise episódica de tornados em Santa Catarina: Caracterização sinótica e mineração de dados. $2003 \mathrm{~b} .223$ p. Dissertação (Mestrado em Sensoriamento Remoto) Instituto Nacional de Pesquisas Espaciais, São José dos Campos, 2003 . 
MOnTEIRo, C. A. F. O clima e a organização do espaço no Estado de São Paulo: problemas e perspectivas. São Paulo: IGEOG/USP, 1976. (Série Teses e Monografias, n. 28).

MONTEIRO, C. A. F. A dinâmica e as chuvas no Estado de são Paulo. Rio Claro: UNESP, Ageteo, 2000 , versão 1.0. CD-ROOM.

NACCARATO, K. P. et al. Lightning activity over large urban areas of Southeastern Brazil. In: International Conference on Atmospheric Electricity, 12., 2003, Versailles, França. Proceedings... Versailles: International Commision on Atmospheric Electricity, 2003, v. 1, p. 67-70.

NACCARATO, K. P. Análise das características dos relâmpagos na região sudeste do Brasil. 2006. 362 p. Tese (Doutorado em Geofísica Espacial) - Instituto Nacional de Pesquisas Espaciais, São José dos Campos, 2006.

NIMER, E. Climatologia do Brasil. Rio de Janeiro: IBGE. 1979.

ORVILLE, R. E. et al. Enhancement of cloud-to-ground lightning over Houston, Texas. Geophysical Research Letters, v. $28, \mathrm{n}$. 13, p. 2597-2600, July 2001.

PEREIRA FILHO, A. J. et al. Enchentes na região metropolitana de São Paulo: aspectos de mesoescala e avaliação de impactos. In: Congresso Brasileiro de Meteorologia, 13, 2004 , Fortaleza, Brasil. Proceedings... Rio de Janeiro: Sociedade Brasileira de Meteorologia, 2004 , CD-ROM.

PINTO, O. Jr.; PINTO, I. R. C. A. Tempestades e relâmpagos no Brasil. São José dos Campos: Instituto Nacional de Pesquisas Espaciais, 2000.

PINTO, I. R. C. A. et al. Urban effect on the characteristics of cloud-to-ground lightning over Belo Horizonte - Brazil. Annales Geophysicae, v. 22, n. 2, p. 697-700, 2004.

PORTUGAL. Instituto de Meteorologia (IP - PORTUGAL). Detectores de raios.

Disponível

em: <http://www.meteo.pt/pt/areaeducativa/fenomenos_meteorologicos/ trovoadas/index.html>. Acesso em: 8 jan. 2009.

SÃo PAULO. Portal do Governo do Estado de São Paulo. Uma potência chamada são Paulo. Disponível em: <http://www.saopaulo.sp.gov.br/saopaulo/>. Acesso em: 8 jan. 2009.

SILVA DIAS, M. A. F. Sistemas de mesoescala e previsão de tempo a curto prazo. Revista Brasileira de Meteorologia, 2, 1987. $133-150$.

SORIANO, L. R.; PABLO, F. Effect of small urban areas in central Spain on the enhancement of cloud-to-ground lightning activity. Atmospheric Environment, v. 36, n. 17, p. 2809-2816, June 2002.

SOUZA, R. Raios, relâmpagos e trovões. Universidade Federal do Pará, Belém, 2002 . Disponível em: <http://www.ufpa.br/ccen/fisica/aplicada/formac.htm>. Acesso em: 2 fev. 2009.

UNESP - UNIVERSIDADE ESTADUAL PAULISTA. INSTITUTO DE PESQUISAS METEOROLÓGICAS. Banco de dados. Bauru, 2007.

UFRRJ - UNIVERSIDADE FEDERAL RURAL DO RIO DE JANEIRO. INSTITUTO DE TECNOLOGIA. Riscos dos raios. Disponível em: <http://www.ufrrj.br/institutos/it/de/acidentes/raios.htm>.

Acesso em: 23 fev. 2009.

WESTCOTT, N. E. Summertime cloud-to-ground lightning activity around major midwestern urban areas. Journal of Applied Meteorology, v. 34, n 7, p. 1633-1642, July 1995. 